DOSSIER

MARION POLGE

ERFI, université de Montpellier I

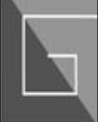

\title{
Les stratégies entrepreneuriales de développement
}

\section{Le cas de l'entreprise artisanale}

Cet article propose d'étudier la notion de stratégie de développement de l'entreprise artisanale en tant que processus entrepreneurial. L'entreprise artisanale est un lieu de valorisation du métier à partir de la maîtrise qu'en détient le dirigeant. Les aspirations à une stratégie de croissance sont limitées en raison des risques de dénaturation des savoir-faire et des compétences distinctives. Or, la stratégie de développement correspond à un déploiement ou une expansion d'activité. Quelles alternatives dans ces conditions pour le développement de l'entreprise artisanale? 
L es notions d' " entreprise artisanale » et de « stratégie entrepreneuriale de développement » sont-elles conciliables?

L'entreprise artisanale est par définition une toute petite entreprise (TPE) ${ }^{1}$ valorisant un métier à partir de la maîtrise qu'en détient son dirigeant. Les aspirations à une stratégie de croissance sont limitées : les actes de délégation consécutifs à la croissance risquent de dénaturer les savoir-faire et les compétences distinctives. Or, la stratégie de développement correspond à un déploiement ou une expansion d'activité (Louart, 1999). Quels sont les enjeux, dans ces conditions, pour le développement de l'entreprise artisanale?

Cette recherche propose d'étudier la notion de stratégie de développement pour l'entreprise artisanale avec l'axe d'approche suivant: le développement peut être assimilé à un processus entrepreneurial dans la mesure où il est engagé par une volonté d'exploitation d'opportunité et sur des changements profonds de système organisationnel, de création de valeur et d'innovation (Verstraete et Fayolle, 2005²). La notion de processus entrepreneurial signifie que la stratégie de développement prend son sens dans son lancement et surtout dans son évolution. "L'évolution même constitue le sens » (Hernandez et Marco, 2006) Compte tenu des caractéristiques de l'entreprise artisanale, quelle forme peut prendre cette stratégie entrepreneuriale?
Deux résultats ont été obtenus :

- un récapitulatif des différentes stratégies de développement et de leurs conditions d'apparition;

- une grille d'analyse permettant de comprendre la logique sous-jacente au parcours du dirigeant;

Cette thématique de travail a été choisie par les membres du club des dirigeants du Languedoc-Roussillon qui représente le creuset méthodologique de cette recherche. Initiée par la profession voici quatre ans, la création de ce $C l u b$ est novatrice à plusieurs titres.

$\mathrm{Du}$ point de vue de la genèse, en premier lieu, un « dispositif global d'accompagnement des dirigeants à l'approche marché » a été mis en place par l'Institut supérieur des métiers $\left(\mathrm{ISM}^{3}\right)$, en réponse à la demande formulée par les organisations professionnelles de l'Artisanat. L'originalité réside en second lieu dans le mode opératoire de la démarche: la méthodologie s'appuie sur une interaction continue avec le terrain. Enfin, l'objectif final vise à conduire les participants à comprendre les stratégies de positionnement pour les entreprises artisanales face à l'évolution des métiers et des environnements. L'accession à une vision entrepreneuriale et stratégique de l'entreprise artisanale apparaît comme un troisième élément novateur.

La présentation de notre travail s'ordonne en trois points: le cadre de la recherche et les axes de la problématique, puis la métho-

1. Sont définies comme artisanales, les entreprises de moins de dix salariés exerçant un métier de production, de transformation ou de service inscrites au registre des métiers.

2. Les auteurs retiennent quatre paradigmes fondamentaux pour définir l'entrepreneuriat: paradigme de l'opportunité, de la création d'une organisation, de l'innovation et de la création de valeur. Nous verrons que la stratégie entrepreneuriale de développement les mobilise successivement dans ses différentes formes.

3. Organisme français chargé d'élaborer des outils d'accompagnement et de formation pour les petites entreprises artisanales. 
dologie de la démarche, et enfin les commentaires des résultats obtenus.

\section{LE CADRE DE LA RECHERCHE : UNE ANALYSE COLLECTIVE DE LA STRATÉGIE ENTREPRENEURIALE DE DÉVELOPPEMENT}

Ce travail s'inscrit dans le cadre d'une recherche collaborative (David, 2000) dans un milieu particulier (Huberman et Miles, 1994) : le club. Sa finalité est de coconstruire des outils d'aide à la décision stratégique grâce à la coparticipation des membres du club au choix des thèmes de travail et aux méthodes adoptées. La problématique a progressivement émergé de ce contexte.

\section{La coconstruction comme fondement académique}

Le terme de coconstruction désigne ici une démarche d'implication conjointe d'acteurs. Les membres du club, artisans, universitaires et institutionnels s'impliquent ensemble en apportant chacun leurs compétences dans le programme de recherche. Deux niveaux de coconstruction sont à considérer: la création de la connaissance et l'amélioration des résultats produits.

La création de la connaissance se fait dans le respect des fondements constructivistes (Le Moigne, 1990) et vise à traduire une représentation du réel prenant en compte la complexité des phénomènes et les effets d'apprentissage en double boucle (Argyris et Schön, 1978), c'est-à-dire une remise en question des processus cognitifs. Les outils coconstruits n'ont pas une ambition universelle. Leur portée se limite aux entreprises similaires en taille, en logique d'action et en environnement.

L'affinement de l'outil s'opère également dans la coconstruction (Argyris et al., 1985).

Une première restitution des résultats fait l'objet de débats, puis sont réalisés des ajustements et des applications personnalisées. Pour finir, une dernière version est livrée au groupe qui décide de l'approuver ou de réviser à nouveau le travail. Comme l'indiquent les travaux de N. Claveau et F. Tannery $(2002)^{4}$, la conception d'artefacts se met en place suivant une logique " spiralée » inscrite dans une démarche ingénierique. La spirale se dessine tout au long des étapes de travail avec le terrain.

\section{La coparticipation comme fondement opératoire}

Une dizaine de chefs d'entreprises artisanales installés sur l'ensemble de la région Languedoc-Roussillon sont associés à ce projet. Ils travaillent dans les secteurs majeurs de l'artisanat: bâtiment et métiers de bouche pour l'essentiel.

La démarche engagée avec eux est à la fois participative et prospective (Barrand et Goy, 2005). Dans ce sens, les questions traitées et le mode d'animation du groupe sont déterminés collectivement. Chacun se découvre progressivement, apprend à se connaître et à mieux comprendre ce que l'autre exprime ou attend (de La Ville, 2001, 2003). Compte tenu de la durée assez

4. La notion d'artefact est employée par les auteurs dans le sens de projet de connaissance et de contexte commun (selon Simon, 1991). 
longue de chaque rencontre ${ }^{5}$ et du programme dans son ensemble, le chercheur peut difficilement se maintenir en position de neutralité d'enquêteur (Kitzinger et Barbour, 1999).

Le construit collectif dégage des aspects thématiques et comportementaux qui influencent la nature des outils (Moscovici, 1984 ; Roy, 2000). Le groupe voit se dessiner un processus itératif d'assimilation et d'analyse des informations débouchant sur des outils concertés.

Ce lent processus met en lumière les biais perceptuels qui existent entre universitaires et artisans. Il pousse à parfaire les explications et à être précis dans les appréciations. Cette volonté d'adéquation entre deux mondes différents en culture et en mode d'expression doit aboutir à des outils reflétant la réalité du terrain.

\section{L'émergence progressive de la problématique}

Le développement en tant que stratégie entrepreneuriale a été peu étudié en petite entreprise.

D'un côté, sont observés certains problèmes particuliers liés à la croissance (Godemer, 2002), d'un autre côté, ont été étudiées plus récemment les entreprises en situation de forte croissance (Julien et al., 2003). Le questionnement qui intéresse les membres du club est différent. Il peut s'articuler autour de deux idées:

- Quand, comment, pourquoi et jusqu'où, la croissance peut-elle être envisagée?
- Le rejet de la croissance signifie-t-il que le dirigeant refuse de s'engager dans une stratégie entrepreneuriale de développement?

G. Koenig (1990) donne un éclairage en caractérisant le développement selon deux formes: autonome (projets internes) et concerté (contractualisé). Cette approche d'ordre général est-elle valable pour les entreprises artisanales?

Pour diagnostiquer la situation des entreprises, le travail de recherche a été adossé aux travaux de l'ERFI (Marchesnay, 1994,1995; Fourcade et Torres, 2001). À partir de la grille d'analyse du système de gestion en petite entreprise (Marchesnay, 1995), nous avons pu structurer la problématique selon les trois axes suivants (voir figure 1):

- Entre désencastrement et encastrement du dirigeant

Le dirigeant oscille entre ces deux tendances (Granovetter, 1995) à la fois opposées et complémentaires. La petite entreprise dispose d'un potentiel de ressources mobilisables faible et de compétences ciblées. L'engagement dans une stratégie entrepreneuriale de développement se fait par un double mouvement: d'un côté, un renforcement d'identité dont seul le dirigeant à la clé (Picard et Thévenard-Puthod, 2004) ; et d'un autre côté, un renoncement d'autonomie par le partage partenarial. Dans sa logique d'action, le dirigeant artisan cherche comment utiliser au mieux son encastrement pour se singulariser de ses confrères.

5. La difficulté méthodologique se situe essentiellement dans la richesse informationnelle et son amplification rapide. L'analyse des données se heurte à la lecture de la complexité et d'une dynamique grandissantes des phénomènes à étudier (Flick, 2001). 
- La combinaison tradition-innovation au cœur des savoir-faire

L'entreprise artisanale évolue aujourd'hui derrière une fragile alchimie alliant la tradition et l'innovation. La notion de métier reste centrale (Richomme, 2001; SchiebBienfait, 2003), mais elle recouvre une réalité complexe: comment faire évoluer les compétences, adhérer et/ou anticiper les attentes des clients tout en préservant les savoir-faire?

Cette question vitale confronte le dirigeant à des problèmes entrepreneuriaux (quel engagement stratégique?), opérationnels (quels choix d'activités?), organisationnels (qui solliciter ou avec qui s'engager?).

\section{- La reconnaissance territoriale}

Le territoire s'entend ici au sens géographique et stratégique. Le périmètre géographique permet de caractériser certaines formes d'identité territoriale, des structures institutionnelles, des modes réglementaires et la proximité physique des confrères. Le territoire stratégique ne se superpose pas au précédent. Il concerne les relations stratégiquement fortes pour l'entreprise sans pour cela être géographiquement proches.

Quel que soit le sens qui lui est donné, le territoire intervient comme un levier relationnel pour créer des axes de développement (Suire, 2004; Echols et Wenpin, 2005). C'est la forme de reconnaissance qui permettra de comprendre le rôle donné au territoire dans la stratégie entrepreneuriale de développement.

Les trois axes de la problématique permettent de dégager une thématique de travail autour de la notion de développement en essayant d'identifier des approches plus spécifiques au domaine de l'artisanat que l'on peut ainsi formuler: Quelle(s) stratégie(s) entrepreneuriale(s) de développement(s) pour les entreprises artisanales? Le choix puis l'étude de la problématique ont été faits dans le cadre du club qui se

Figure 1 - Les axes de la problématique

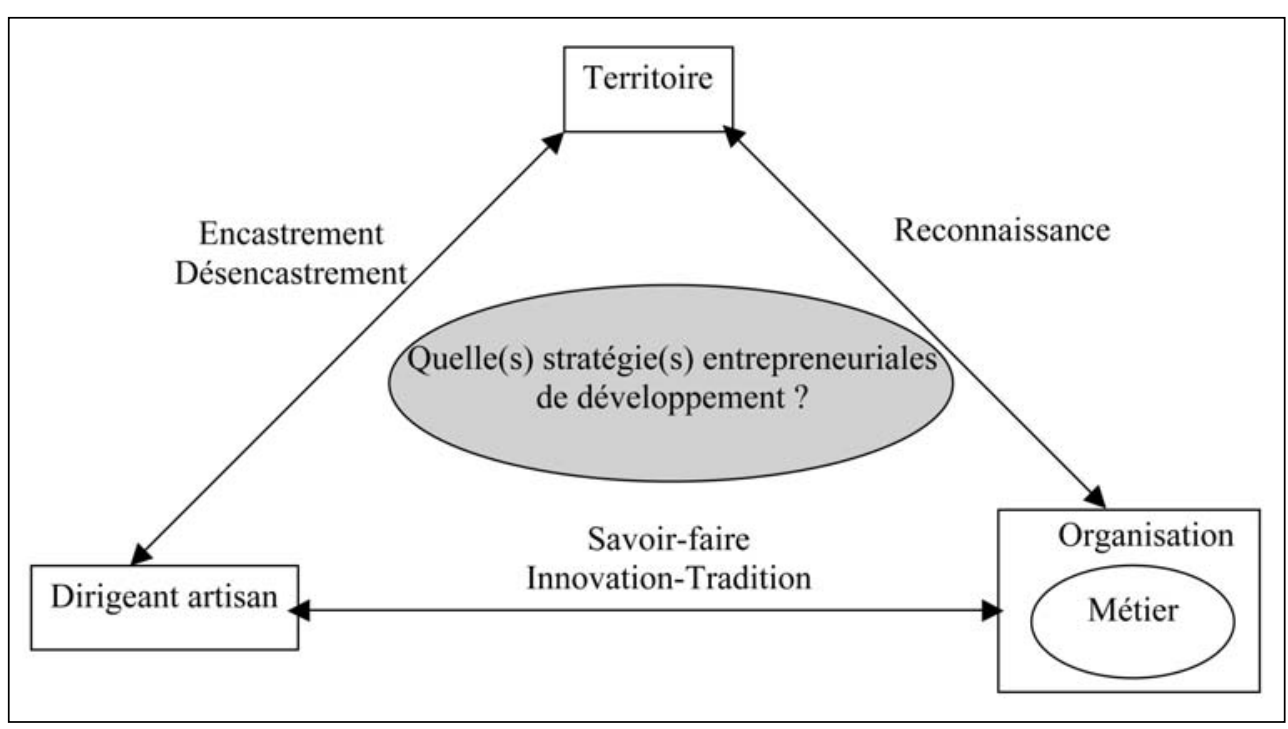


présente comme une méthodologie originale que nous allons décrire.

\section{LA MÉTHODOLOGIE COMBINÉE DE LA DÉMARCHE}

La méthodologie adoptée pour animer le club constitue une forme détournée d'observation participante dans la mesure où l'on suscite la confiance des enquêtés à partir d'une action commune afin de concevoir les outils d'aide à la décision.

\section{Le déroulement des échanges}

Concrètement, la conduite du club se déroule en mixant des entretiens et des séminaires répartis sur l'année universitaire formant le socle des cycles de travail.

Les quatre séminaires annuels sont l'occasion de circonscrire les thématiques et de prendre du recul sur les outils proposés.

Les deux entretiens individuels menés dans chaque cycle se glissent entre les séminaires collectifs; ils se déroulent dans l'entreprise ou sur les chantiers. Le face à face approfondi libère les dirigeants sur certains sujets délicats et facilite la compréhension $\mathrm{du}$ quotidien de l'entreprise. Cette technique permet de recueillir des données semblables à celles habituellement obtenues avec les études de cas, mais elle prend tout son relief en confrontation avec les séminaires précédents et suivants. En servant de relais entre les séminaires, les entretiens sont donc l'occasion de renforcer la dimension longitudinale et approfondie de la recherche.

Ce travail porte plus sur le processus que sur le contenu, c'est-à-dire le parcours d'évolution d'une ou plusieurs variables à travers le temps (Van de Ven, 1992; Monge, 1990). Le mixage entre les séminaires et les entretiens permet de comprendre la logique d'évolution de la variable « prise de décision stratégique »(Grenier et Josserand, 1999).

\section{La méthodologie de conception des outils}

Les questions relatives aux données sont posées à deux niveaux: la nature du recueil orientant la portée de l'outil et le traitement des données.

\section{Le recueil}

Le temps est indissociable de cette recherche longitudinale pour laquelle a été retenue une approche chronologique, c'està-dire d'observation d'un phénomène qui " existe en dehors de la recherche " (Forgues et Vandangeon-Derumez, 1999), de son processus d'émergence et de consolidation.

Cette posture fournit des données primaires à la fois rétrospectives et en temps réel. L'un des principaux biais des narrations a posteriori se rencontre avec la tentative de rationalisation de l'enquêté (Golden, 1992). Or, il est possible de procéder à des recoupements d'informations entre les différents entretiens et/ou séminaires (Yin, 1990).

\section{Le traitement des données}

L'esprit de l'analyse se rapproche des propositions de A. M. Huberman et M. B. Miles (1994) puisqu'il s'agit de repérer l'évolution d'un phénomène en établissant une relation temporelle entre les variables. Cette idée, propre aux études longitudinales peut être opérationnalisée de différentes manières. Nous avons privilégié des analyses qualitatives comparatives (Chanson et al., 2005) systémiques soulignant les processus d'évolution et les liens de causalités 
entre les variables qui les impulsent. Le choix de traitement prépare la mise en place d'un outil insistant sur les liens de causalité réciproque entre les variables telles que se les représente le dirigeant.

\section{RÉSULTATS ET DISCUSSION}

Sur le plan instrumental, différentes formes de stratégies de développement ont été identifiées ainsi que leurs liens. Une grille d'analyse permettant de repérer les parcours qui leur sont associés a été mise en place. Ces résultats vont respectivement être présentés.

\section{Les stratégies entrepreneuriales de développement en entreprise artisanale}

Deux choix s'offrent au dirigeant: celui de la croissance, c'est-à-dire le développement interne ou bien celui $d u$ développement par "non-croissance ». Chacun implique des changements difficilement réversibles et peuvent donc être considérés comme entrepreneuriaux. D'une certaine manière, "l'activité artisanale repose sur l'activité entrepreneuriale » (Paturel et Richomme, 2007) dans la mesure où le dirigeant crée de la valeur par la stratégie de développement en s'appuyant sur sa particularité.

Le développement interne est l'orientation perçue comme la plus délicate: induisant une augmentation du volume d'activité, ce mode de développement implique des changements d'effectif, de méthodes de travail, la multiplication des activités, etc. Il requiert un remaniement de l'organisation existante avec une structuration progressive et une réattribution des tâches. En artisanat, ce choix stratégique soulève des questionnements bien particuliers du fait de l'implication du dirigeant dans l'activité produc- tive, de la proximité relationnelle des membres de l'entreprise et de la nature du métier. Le rôle de dirigeant se transforme et intègre de plus en plus de responsabilités managériales, de gestion et d'animation auxquelles il n'est pas nécessairement préparé. De nombreux dirigeants, conscients des difficultés de gestion liées à cette orientation, cherchent à l'éviter.

À ces bouleversements s'ajoute une remise en question des compétences distinctives de l'entreprise dont le métier ne peut plus s'exercer à l'identique. La réaffectation des ressources stratégiques nécessite une reconstruction de la capacité dynamique.

Le développement par non-croissance traduit une absence d' «effet taille». Les caractéristiques de l'organisation sont revisitées et ses fondements sont revus selon les acteurs impliqués dans la trajectoire stratégique et selon les projets de l'entreprise. Deux situations se dégagent: l'entreprise se développe par le partenariat, et nous parlerons de développement externalisé, ou bien le dirigeant choisit le renouvellement et la créativité continus, forme qualifiée de développement incrémental.

Le développement externalisé consiste à diffuser l'accroissement du volume d'activité à travers un réseau de confrères. Ensemble, les dirigeants engagent un processus collectif de construction d'une nouvelle organisation (voir les travaux de Verstraete et Fayolle, 2005) plus ou moins ouverte, suscitant un double effet: chacun se recentre sur son cœur de métier et renforce sa position, tandis que le réseau élargit le domaine de compétences.

Cette stratégie aboutit à une forme de travail répartie entre plusieurs entreprises qui se coordonnent, s'ajustent, codécident, de sorte que le périmètre de l'organisation 
dépasse celui de l'entreprise. Le développement externalisé présente plusieurs options: l'une formalisée par l'intermédiaire d'un hypo-groupement et d'alliances, et l'autre non formalisée avec des ententes ponctuelles, ou un réseau de partenaires.

Les données que nous avons recueillies semblent montrer un engouement pour cette forme de développement. Il est toutefois à noter que les artisans sont méfiants sur deux points:

- la contractualisation du partenariat: ils privilégient des rapprochements informels basés sur la confiance professionnelle et le lien affectif. L'engagement écrit leur paraît plus rigide et plus risqué;

- les limites du partage: les rapprochements se font souvent sur des activités connexes et certains craignent la perte de savoir ou l'absorption par un concurrent.

Le développement incrémental est synonyme de changement continu. L'entreprise est mise au service de l'innovation et de la créativité (Verstraete et Fayolle, 2005). Les systèmes de décision, d'organisation, de production évoluent continuellement. Cette forme de développement est fréquente dans les travaux par projets (comme les travaux de grande ampleur dans le bâtiment), l'artisanat d'art, ou encore les activités technologiquement innovantes (les technologies liées à la communication pour les électriciens). L'entreprise est mise dans une posture d'apprentissage permanent et de remise à plat des processus. Particulièrement appréciée par les membres du club, cette forme de développement correspond aux aspirations des artisans, car elle utilise le lien « tradition-innovation » dans le métier pour soutenir le processus entrepreneurial.
Le tableau 1 présente les conditions dans lesquelles se rencontrent ces trois formes de développement. Le contraste entre les différentes stratégies entre-preneuriales est marqué à tous les niveaux.

Le contexte. Le développement interne est privilégié lorsque le dirigeant est méfiant à l'égard des acteurs de son environnement. Des perspectives plus favorables le poussent vers le développement par non-croissance.

Les contraintes. Le développement interne effraie du fait de la perte de maîtrise de la réalisation du métier. Le développement partenarial présente les mêmes contraintes, mais en dehors du périmètre de l'entreprise. La perception du risque encouru est moins élevée. Avec le développement incrémental, la demande devient plus exigeante et la prestation plus délicate.

Les implications. Le développement par croissance donne un sentiment de sécurité et de protection à la fois externe, mais aussi interne (moins de dépendance visà-vis des salariés expertisés dans un métier). L'externalisation du développement permet de recentrer l'activité et de préserver la flexibilité, tandis que le développement incrémental soutient une stratégie de singularité.

\section{Les relations entre les trois stratégies entrepreneuriales}

\section{Non exclusivité}

Les trois stratégies identifiées peuvent coexister dans l'entreprise. Les dirigeants mixent régulièrement les différentes alternatives. Simplement, ils activent davantage l'une des orientations à certains moments, mettant les autres en sommeil ou au second plan. 
Tableau 1 - Conditions d'apparition des trois formes de développement

\begin{tabular}{|c|c|c|c|}
\hline & Développement par & \multicolumn{2}{|c|}{ Développement par non-croissance } \\
\hline & $\begin{array}{l}\text { Développement interne } \\
\text { (recrutement/changement } \\
\text { de local/diversification) }\end{array}$ & $\begin{array}{l}\text { Développement partenarial } \\
\text { (franchises/partenariats/ } \\
\text { alliances) }\end{array}$ & $\begin{array}{l}\text { Développement } \\
\text { incrémental } \\
\text { (création de nouveaux } \\
\text { produits, } \\
\text { nouvelles techniques, } \\
\text { utilisation d'informations } \\
\text { nouvelles) }\end{array}$ \\
\hline Contexte & $\begin{array}{l}\text { - Environnement hostile } \\
\text { ou non fiable } \\
\text { - Facilité d'imitation } \\
\text {-Volonté de maîtrise d'un } \\
\text { savoir-faire technique ou } \\
\text { commercial } \\
\text { - Volonté d'indépendance }\end{array}$ & $\begin{array}{l}\text { - Environnement fiable } \\
\text { - Recherche de } \\
\text { compétences } \\
\text { complémentaires } \\
\text { - Maîtrise d'un savoir-faire } \\
\text { rare } \\
\text { - Volonté de partage }\end{array}$ & $\begin{array}{l}\text { - Débouchés importants } \\
\text { - Produits à dominante } \\
\text { innovante et créative } \\
\text { - Durée de vie des } \\
\text { produits } \\
\text {-Volonté d'apprentissage } \\
\text { et de défis } \\
\text { - Communication }\end{array}$ \\
\hline Contraintes & $\begin{array}{l}\text { - Géographique (lieu } \\
\text { d'implantation, volume de } \\
\text { marché) } \\
\text { - Financières } \\
\text { (endettement/soutien du } \\
\text { banquier) } \\
\text { - Recrutement/formation } \\
\text { des recrutés } \\
\text { - Organisation de } \\
\text { l'activité: délégation des } \\
\text { compétences-clés }\end{array}$ & $\begin{array}{l}\text { - Dépendance des } \\
\text { partenaires } \\
\text { - Décisions et qualité liée } \\
\text { aux partenaires }\end{array}$ & $\begin{array}{l}\text { - Géographiques: } \\
\text { débouchés parfois hors } \\
\text { territoire } \\
\text { - Compétences rares: } \\
\text { difficiles à } \\
\text { trouver/formation longue }\end{array}$ \\
\hline Implications & $\begin{array}{l}\text { - Autonomie } \\
\text { - Contrôle } \\
\text { - Inquiétudes en gestion }\end{array}$ & $\begin{array}{l}\text { - Flexibilité } \\
\text { - Concentration sur le } \\
\text { métier } \\
\text { - Produits plus spécifiques }\end{array}$ & $\begin{array}{l}\text { - En marge de la } \\
\text { concurrence } \\
\text { - Mobilité } \\
\text { interne/externe/de prise de } \\
\text { décision }\end{array}$ \\
\hline
\end{tabular}

\section{Dynamique}

Les quatre années d'existence du club donnent suffisamment de recul pour observer la dynamique. Les dirigeants peuvent modifier leur trajectoire très rapidement (dans un délai de deux à trois semaines) soit par constat d'erreur, soit à la suite de modifications contextuelles.

Il existe donc des mouvements de balancier que présente la figure 2 ci-après. L'une des limites actuelles de cette recherche est de ne pas parvenir à repérer 
Figure 2 - Les liens entre les stratégies entrepreneuriales de développement

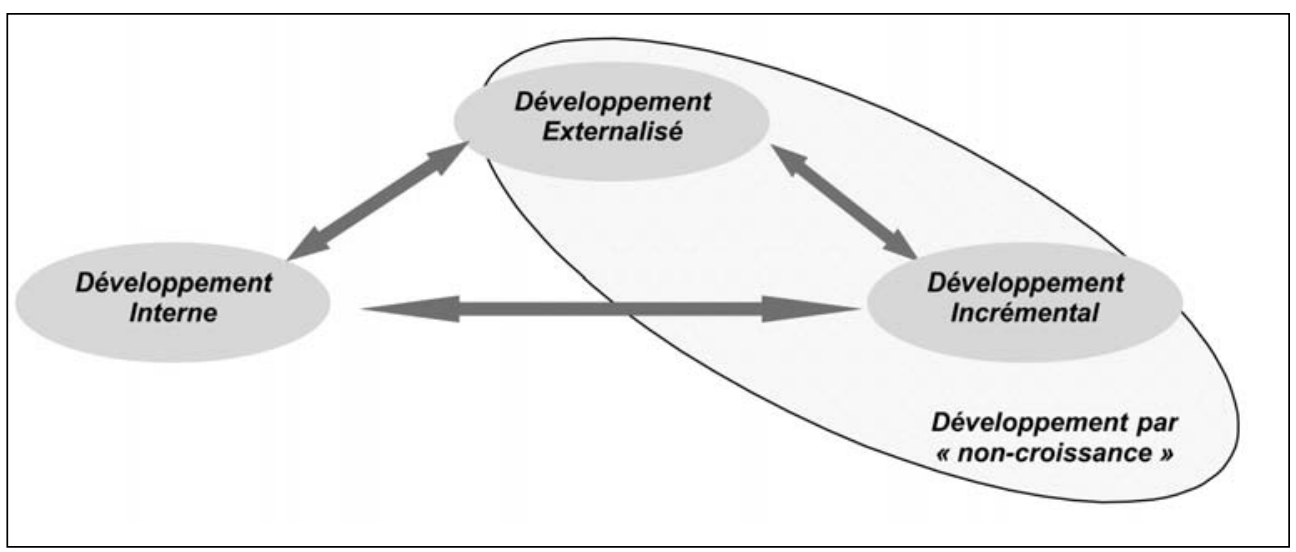

le rythme et les motivations de basculement de ce balancier.

Les premiers résultats concernent la caractérisation des stratégies entrepreneuriales de développement. Le dirigeant suit un parcours qui l'amène à choisir alternativement l'une des formes de développement. L'outil mis en place pour le guider va maintenant être présenté.

\section{Le système de construction des stratégies entrepreneuriales de développement}

L'issue du travail du club doit pouvoir bénéficier à d'autres entreprises souhaitant se développer. Nous avons vu les limites de ce type de méthode de recherche en matière de généralisation. Dans une approche prospective, il a donc été décidé que le club mettrait en place des grilles d'analyse d'autodiagnostic et d'identification des parcours cognitifs suivis par le dirigeant (GouxBoudiment, 2000).

La grille d'analyse des stratégies entrepreneuriales de développement en artisanat s'articule autour de deux ensembles de questions: le premier est lié au dirigeant et à sa relation au métier; le second est relatif au développement proprement dit de l'entreprise et à son contexte territorial.

\section{L'artisan et son métier (voir tableau 2)}

Le profil du dirigeant: selon le parcours, les succès, les échecs, chacun envisage différemment sa vie dans l'entreprise. Il s'agit aussi bien de repérer les caractéristiques objectives du responsable de l'entreprise que de comprendre son ressenti en tant qu'individu.

L'approche du métier: la question posée ici est celle du positionnement de l'artisan-dirigeant dans sa propre organisation. Avec l'accroissement du volume de l'activité, beaucoup d'artisans s'écartent de l'exercice direct du métier pour se consacrer plutôt à la conduite de leur entreprise, ce qui n'est pas sans incidence sur l'impulsion entrepreneuriale donnée au développement.

La stratégie de 1'entreprise sera très largement déterminée par ces deux critères (pro- 
Tableau 2 - Variables d'identification de l'artisan et de son métier

\begin{tabular}{|c|l|}
\hline \multirow{4}{*}{$\begin{array}{c}\text { Le profil } \\
\text { du dirigeant }\end{array}$} & $\begin{array}{l}\text { En tant que responsable d'une entreprise artisanale: } \\
- \text { Qui est le décideur (dirigeant/conjoint/collaborateur)? } \\
- \text { Y a-t-il une indépendance financière? } \\
\text { - Quelle autonomie pour les salariés?: } \\
\text { Pour faire plus de commercial/pour plus innover/pour améliorer la qualité de vie }\end{array}$ \\
\cline { 2 - 3 } & $\begin{array}{l}\text { En tant qu'individu-dirigeant: } \\
\text { - Volonté d'entreprendre, de relever des défis } \\
\text { Nouveaux produits/nouvelle clientèle/travailler avec d'autres artisans/sortir de son } \\
\text { territoire } \\
\text {-Volonté de progresser dans le métier qui peut être liée: } \\
\text { Au savoir-faire, au management, aux techniques de gestion }\end{array}$ \\
\hline \multirow{2}{*}{$\begin{array}{c}\text { Le métier } \\
\text { du dirigeant }\end{array}$} & $\begin{array}{l}\text { Quel est le métier du dirigeant artisan? : } \\
- \text { Chef d'entreprise } \\
\text { Beaucoup de temps accordé à l'activité commerciale } \\
\text { Analyse régulière des documents d'aide au pilotage } \\
\text { Plus de temps hors de l'entreprise que sur les chantiers ou à l'atelier } \\
- \text { Animateur d'équipe } \\
\text { Dans l'entreprise en tant que chef d'équipe } \\
\text { Avec les partenaires (confrères/clients/fournisseurs, etc.) } \\
- \text { Valorisateur d'un savoir-faire } \\
\text { Recherche de la rareté et de l'excellence } \\
\text { Recherche de perfectionnement technique } \\
\text { Recherche d'innovation }\end{array}$ \\
\hline
\end{tabular}

fil du dirigeant/approche du métier), qui constituent l'axe fondateur des choix de développement.

\section{Le développement de l'entreprise et son environnement territorial (voir tableau 3)}

Le développement de l'entreprise. Les deux derniers paragraphes ont été consacrés à sa description. Nous ne reviendrons pas sur ce point.

L'environnement territorial. L'environnement territorial s'ordonne selon trois catégories : le réseau de partenaires, les relations commerciales (y compris la concurrence) et les institutions.
Les artisans connaissent relativement bien leur environnement partenarial par l'intermédiaire de leurs organisations professionnelles, des salons, des clients ou encore du voisinage. Les réseaux qu'ils activent deviennent de véritables creusets pour le développement externalisé. Ils donnent des indications de confiance, de domaine de compétences et d'orientation des confrères. De l'environnement commercial et plus largement concurrentiel se dégagent des sentiments de protection ou au contraire d'hostilité. Ils vont arbitrer l'engagement vers l'une des trois formes de développement. Enfin, l'environnement 
Tableau 3 - Variables d'identification du développement et de l'environnement territorial

\begin{tabular}{|c|c|}
\hline $\begin{array}{c}\text { Stratégies entrepreneuriales } \\
\text { de développement }\end{array}$ & $\begin{array}{l}\text { Développement par croissance: développement interne } \\
\text { Développement par non croissance: } \\
\text { - Développement partenarial } \\
\text { - Développement incrémental }\end{array}$ \\
\hline \multirow{3}{*}{$\begin{array}{l}\text { Environnement } \\
\text { territorial }\end{array}$} & $\begin{array}{l}\text { Environnement partenarial: } \\
\text { Son existence est... } \\
\text { - Liée à des structures formelles/une démarche émergente } \\
\text { - Proactive/réactive } \\
\text { - Déterminante/accessoire }\end{array}$ \\
\hline & $\begin{array}{l}\text { Environnement concurrentiel: } \\
\text { - Relations de pouvoir } \\
\text { - Hostilité/complémentarité/soutien } \\
\text { - Sentiment de protection: singularité } \\
\text { - Sentiment de méfiance: fragilité/incertitude/imitation }\end{array}$ \\
\hline & $\begin{array}{l}\text { Environnement institutionnel: } \\
\text { - Dirigeant impliqué/peu impliqué } \\
\text { - Nature de l'institution/situation territoriale } \\
\text { - Rôle dans l'institution/attentes } \\
\text { - Répercussions dans la conduite quotidienne de l'entreprise }\end{array}$ \\
\hline
\end{tabular}

institutionnel laisse aux artisans du club une impression de perplexité: malgré une implication régulière et insistante, ils ne parviennent pas à repérer de réels appuis.

Les choix de développement et la perception de l'environnement territorial ont été retenus pour soutenir le second axe de la grille d'analyse.

\section{Proposition d'une grille d'analyse d'autodiagnostic}

Les deux axes dirigeant/métier et développement/environnement se combinent de façon interdépendante:

- la stratégie entrepreneuriale de développement est conditionnée par le parcours du dirigeant et sa conception du métier;
- de manière réciproque, les relations avec les confrères et le dynamisme territorial ont une incidence sur la conception qu'a le dirigeant de son travail et sa disposition à collaborer.

Le système mis en place évolue continuellement, ce qui explique les basculements entre les formes de développement. Ainsi, la stratégie de développement revêt un caractère très changeant, qui la place dans une posture entrepreneuriale, et non dans une stratégie managériale. L'objet du développement en artisanat n'est pas de s'installer dans une démarche linéaire, mais au contraire de modifier la trajectoire selon l'évolution contextuelle interne et/ou externe.

Le croisement des axes permet d'aboutir à la grille d'analyse présentée par la figure 3 . 
Figure 3 - Le système de développement en entreprise artisanale

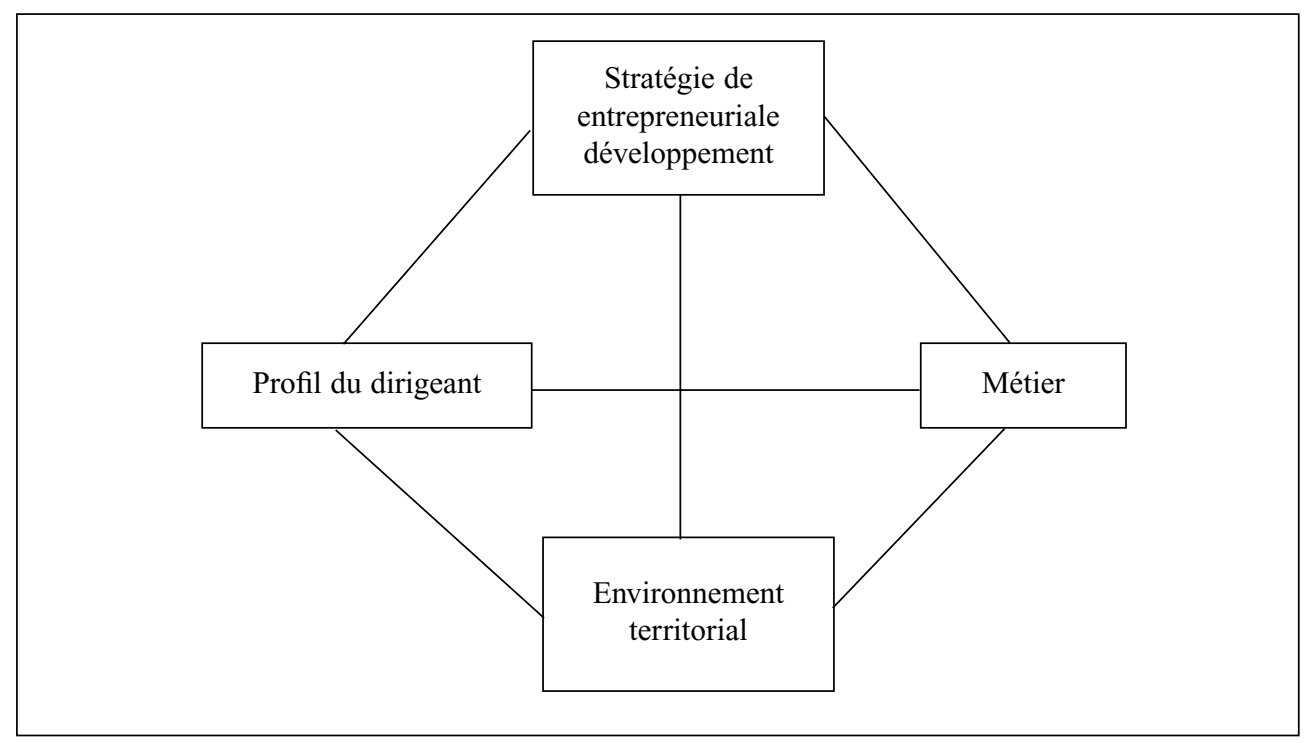

\section{CONCLUSIONS ET IMPLICATIONS}

L'objectif premier de ce travail était de concevoir des outils flexibles et adaptables aux besoins des artisans. La méthodologie retenue a donné satisfaction quant à la nature de la grille présentée et à l'utilisation régulière qui en est faite par les membres du club.

Plus largement, une expérience nationale est actuellement menée. Une version informatisée de la grille d'analyse a été créée et peut être utilisée en accès privé sur le site de l'ISM. Les retours d'expérience des dirigeants internautes sont positifs à deux niveaux: le questionnement proposé les place dans une posture de réflexion et les propositions qui leur sont adressées apportent un éclairage nouveau.

Par ailleurs, le stockage des données effectué lors de leur connexion devrait nous permettre d'affiner nos résultats et d'envisager une généralisation plus rigoureuse.
D'un point de vue académique, il a été possible de mieux cerner la notion de stratégie entrepreneuriale de développement pour les artisans. Contrairement aux premiers constats, l'attachement au métier n'intervient pas comme un handicap. Au contraire, il ouvre des perspectives entrepreneuriales originales qui renforcent la particularité des entreprises de ce secteur. Deux enseignements nous ont particulièrement interpelés. - Tout d'abord, la rapidité du changement de la stratégie. Le processus entrepreneurial n'est pas seulement dans le choix de se développer. Il se trouve également dans la volonté de modifier sans cesse le mode de développement et de constituer des combinaisons nouvelles de formes de développement.

- Ensuite, l'existence d'une forme atypique: le développement incrémental. Son impact sur l'entreprise se traduit par une augmentation des marges et du confort dans 
la vie au travail. Les dirigeants parlent à son propos de « plaisir » dans la créativité, ou encore de partage de la satisfaction. L'artisanat offrirait-il ainsi une nouvelle conception de l'entrepreneuriat du développement?

Cette démarche présente encore certaines limites.

La première tient à la spécificité du tissu local. Des expériences similaires à celle-ci sont actuellement menées dans plusieurs régions françaises. Les premiers résultats montrent des différences significatives dans le comportement stratégique des artisans, comme dans la perception qu'ils ont de leur activité et de leur environnement.

La seconde limite tient à la composition même du club. Les artisans ont été contac- tés par les institutions locales qui les représentent et qui les connaissent. Il s'agit pour l'essentiel de dirigeants très dynamiques, impliqués dans la vie de leur branche professionnelle. De ce fait, ils sont déjà en attente d'outils, de réflexion et d'échange. On peut dès lors s'interroger sur la généralisation à l'ensemble de l'artisanat régional.

Cette recherche longitudinale se poursuit: dans le club, chaque forme de développement a été étudiée de façon approfondie et a donné lieu à une grille d'analyse ou une série de grilles spécifiques. Entre les clubs, un effort d'échange de connaissances a été entrepris. L'objectif est de parvenir à une convergence plus forte pour accompagner ces entreprises en mutation.

\section{BibliographIE}

Argyris C., Schön D. A., Theory in practice: Increasing professional effectiveness, JosseyBass, 1978.

Argyris C., Putnam R., McLain Smih D., Action Science, Jossey-Bass, 1985.

Barrand J., Goy H., « Les apports de la prospective aux approches cognitives de la stratégie en PME », Revue Internationale PME, vol. 18, n 2, 2005, p. 109-139.

Bootz J. P., « La prospective, un outil de creation de connaissances: perspective cognitive et observation participante », Finance Contrôle et Stratégie, vol. 8, n 3, septembre 2005, p. 5-27.

Chanson G., Demil B. et Lecocq X., « La place de l'analyse qualitative comparée en sciences de gestion », Finance, Contrôle et Stratégie, vol. 8, n 3, septembre 2005, p 29-50.

Claveau N., Tannery F., « La recherche à visée ingénierique en management stratégique ou la conception d'artefacts médiateurs », Questions de méthodes en Sciences de Gestion, chap. 4, Mourgues N. et al., Paris, MES, 2002, p. 121-150.

David A., « La recherche-intervention, cadre général pour la recherche en management? », chapitre 8, Les nouvelles fondations des sciences de gestion, David A., Hatchuel A. et Laufer R., Paris, Fnege, 2000, p. 193-213.

De La Ville V., « L'émergence du projet entrepreneurial: apprentissages, improvisations et irréversibilités », Revue de l'Entrepreneuriat, vol. 1, n 1, 2001, p. 43-59. 
De La Ville V., « D’écrire la stratégie: Réflexions sur la production de récits dans la recherche en management stratégique », Revue Sciences de Gestion, ${ }^{\circ}$ 32, 2003, p. 131-152.

Echols A., Wenpin T., « Niche and performance: the moderating role of network embeddedness », Strategic Management Journal, vol. 26, 2005, p. 219-238.

Forgues B., Vandangeon-Derumez I., " Analyses longitudinales », Méthodes de recherche en management, chap. 15, Thiétart R.A. et al., Paris, Dunod, 1999, p. 422-448.

Fourcade C., Torrès O., « Proximité et stratégies d'internationalisation des PME: le rôle des milieux internationalisants », III Journées de la Proximité, "Nouvelles croissances et territoires », Paris 13-14 décembre 2001.

Godener A., «PME en croissance: peut-on prévoir les seuils organisationnels? », Revue Internationale PME, vol. 15, $\mathrm{n}^{\circ} 1,2002$.

Golden B. R., "The past is the past or is it? The use of retrospective accounts as indicators of past strategy", Academy of Management Journal, vol. 35, n 4, 1992, p. 848-860.

Goux-Boudiment F., "Préface ", Penser le futur. L'essentiel de la prospective et de ses méthodes, Masini E.B., Dunod, 2000.

Granovetter M., «La notion d'embeddedness », L'inscription sociale du marché, Jacob A. et Verin H., L'Harmattan, Paris, 1995, p. 11-21.

Grenier C., Josserand E., « Recherches sur le contenu et recherches sur le processus », Méthodes de recherche en management, chap. 5, Thiétart R.A. et al., Paris, Dunod, 1999, p. 104-136.

Hernandez E-M., Marco L., Entrepreneur et Décision, Eska Editions, 2006.

Huberman A. M., Miles M. B., "Data Management and analysis methods", Handbook of qualitative research, Denzin N. K., Lincoln Y.S., Thousand Oaks, Sage, 1994, p. 428-444.

Julien P. A., avec la coll. de Luc D., Carrier C., Desaulniers L., Martineau Y., Les PME à forte croissance, Presses de l'Université du Québec, 2003.

Kitzinger J., Barbour R.S., Introduction: The challenge and promise of focus groups, Barbour R.S. et Kitzinger J. (Eds), Developping Focus Group Research, Thousand Oaks and London, Sage, 1999.

Koenig G., Management Stratégique, Nathan, 1990.

Koenig G., " Production de la connaissance et constitution des pratiques organisationnelles », Revue de Gestion des Ressources Humaines, n 9, novembre 1993, p. 4-17.

Le Moigne J.L., « Epistémologies constructivistes et sciences de l'organisation », Espistémologies et Sciences de gestion, Martinet A.C., Economica, 1990.

Louart P., « Développement», Encyclopédie de la Gestion et du Management, EGM, Dalloz, 1999.

Marchesnay M., Julien P.A., Les PME, Bilan et perspectives, GREPME, Éd. Economica, Paris, 1994.

Marchesnay M., Management Stratégique, Eyrolles Universités, coll. « Gestion», Paris, 1995. 
Monge P, "Theorical and analytical issues in studying organizational processes", Organization Science, vol. 1, n 4, 1990, p. 406-430.

Moscovici S., "The phenomenon of social representation", Social representations, Farr R.M. et Moscovici S. (éds), Cambridge University Press, 1984.

Paturel R., Richomme K., « Le devenir de l'activité artisanale passe-t-il par l'activité entrepreneuriale? », Revue de l'Entrepreneuriat, vol. 6, $\mathrm{n}^{\circ}$ 1, 2007.

Picard C., Thévenard-Puthod C., « La reprise de l'entreprise artisanale: spécificité du processus et de conditions de sa réussite », Revue Internationale PME, vol. 17, $\mathrm{n}^{\circ} 2,2004$, p. 93-121.

Richomme K., « De la cotraitance au management relationnel: le cas d'une coopérative artisanale du secteur du bâtiment », Revue Internationale PME, vol. 14, $\mathrm{n}^{\circ}$ 2, 2001, p. 109-131.

Roy B., « L'aide à la décision aujourd'hui : que devrait-on attendre? », chap. 6, David A., Hatchuel A. et Laufer R., Les nouvelles fondations des Sciences de Gestion, Paris, Fnege, 2000, p. 141-174.

Schieb-Bienfait N., «L'analyse stratégique à l'épreuve des faits: étude de cas d'une petite entreprise artisanale en mutation », Revue International PME, vol. 16, $\mathrm{n}^{\circ} 12,2003$, p. 133-163.

Simon H.A., Sciences des systèmes-sciences de l'artificiel, Paris, Dunod, 1991.

Suire R., « Des réseaux de l'entrepreneur aux ressorts du créatif: quelles stratégies pour les territoires? », Revue Internationale PME, vol. 17, $\mathrm{n}^{\circ}$ 2, 2004, p 123-144.

Thiétart R.-A.(dir.), Stratégies: actualité et futurs de la recherche, Paris, Vuibert, p. 101-114.

Van de Ven A., "Suggestions for studying strategy process: a research note", Strategic Management Journal, vol. 13 (summer special issue), 1992, p. 169-188.

Verstraete T., Fayolle A., "Paradigmes et entrepreneuriat", Revue de l'Entrepreneuriat, vol. 4, $\mathrm{n}^{\circ} 1,2005$.

Yin Y.K., Case Study Research: design and methods, Thousand Oaks, CA, Sage, 1984, 1990. 